\title{
Locally advanced laryngeal cancer: Total laryngectomy or primary non-surgical treatment?
}

\author{
ALEŠ ČOČEK ${ }^{1,2}$, MILOSLAV AMBRUŠ $^{3}$, ALENA DOHNALOVÁ ${ }^{4}$, MARTIN CHOVANEC $^{2}$, \\ MARTINA KUBECOVÁ ${ }^{3}$ and KATEŘINA LICKOVÁ ${ }^{3}$
}

\author{
${ }^{1}$ ENT Department, Oblastní Nemocnice Příbram, 26101 Př́íbram; ${ }^{2}$ ENT Clinic; ${ }^{3}$ Radiotherapy and Oncology Clinic, \\ Charles University 3rd Medical School and Royal Vinohrady Teaching Hospital, 10034 Prague; ${ }^{4}$ Institute of Physiology, \\ Charles University 1st Medical School and General Teaching Hospital, 12800 Prague, Czech Republic
}

Received January 1, 2017; Accepted June 9, 2017

DOI: $10.3892 / \mathrm{ol} .2018 .8150$

\begin{abstract}
Between January 1997 and December 2013, the Charles University 3rd Medical School and Royal Vinohrady Teaching Hospital Ear, Nose and Throat oncology team treated 185 patients with advanced laryngeal cancer, which, from a surgical perspective, required a total laryngectomy. Overall, $\sim 70 \%$ of these patients $(n=129)$ underwent conventional treatment (i.e., total laryngectomy with post-operative radiotherapy), and $\sim 30 \%(n=56)$ were treated with larynx preservation protocols (including primary radiotherapy, neoadjuvant chemotherapy followed by radiotherapy or chemoradiotherapy, or primary chemoradiotherapy). Patients treated with laryngeal preservation protocols had a 5-year survival probability of $48 \%$, whereas those treated with total laryngectomy and post-operative radiotherapy had a 5-year survival probability of $63 \%$. This difference was not statistically significant. However, patients who underwent primary surgical treatment survived for a significantly longer period $(\mathrm{P}<0.010)$. The sex of the patient did not have a statistically significant impact on patient survival probability. More extensive local disease and more advanced disease stages conferred a lower survival probability, but were not statistically significant; however, a lower survival probability in patients $>70$ years was identified to be statistically significant $(\mathrm{P}<0.010)$. Local disease recurrence and recurrent cervical nodal metastases had a statistically significant impact on the 5-year survival probability $(\mathrm{P}<0.001)$. A step wise Cox regression analysis was used to compare the parameters of sex, patient age, tumor extent, disease stage, choice of primary surgery, local recurrence and cervical nodal recurrence. In
\end{abstract}

Correspondence to: Dr Kateřina Licková, Radiotherapy and Oncology Clinic, Charles University 3rd Medical School and Royal Vinohrady Teaching Hospital, Šrobárova 50, 10034 Prague, Czech Republic

E-mail:katkalic@seznam.cz

Key words: total laryngectomy, laryngeal cancer, radiotherapy, chemotherapy, preservation protocol the first step, local recurrence was selected as the parameter having the greatest effect on survival $(\mathrm{P}<0.001)$; patient age $>70$ years $(\mathrm{P}<0.001)$ was selected in the second step; cervical nodal recurrence $(\mathrm{P}<0.001)$ in the third step; and disease stage $(\mathrm{P}<0.010)$ in the fourth step. Other parameters did not significantly affect survival. The results of the present study confirmed that primary non-surgical treatment is an alternative approach to total laryngectomy, and that an informed patient should determine the treatment approach. The decreased overall survival observed in more extensive tumors suggests that surgical treatment may be a better selection in these cases. Due to increased overall survival, primary non-surgical treatment may be recommended for younger patients. If the patient chooses primary non-surgical treatment, concomitant chemoradiotherapy is recommended. If the patient cannot tolerate cytostatic chemotherapy, radiotherapy alone is recommended.

\section{Introduction}

Total laryngectomy is an established, effective treatment for locally advanced laryngeal cancer (1). However, it is a disfiguring procedure that markedly alters the patient's post-operative quality of life. The results of pilot studies have indicated that primary non-surgical procedures, that is, neoadjuvant systemic cytostatic chemotherapy followed by radiotherapy or concomitant chemoradiotherapy, may be just as effective as surgery (1); this assumes that total laryngectomy is available when conservative treatments fail. The benefit of these procedures lies in the potential to preserve the larynx in a relatively large proportion of patients $(1,2)$. Primary indications for non-surgical treatment (preservation protocols) and total laryngectomy are presently very topical and a consensus has not yet been reached.

Conventional treatment for advanced laryngeal cancer (T3 and T4) with total laryngectomy, or total laryngectomy with post-operative radiotherapy, is associated with significant patient disfiguration (3). Permanent tracheostomy, loss of a natural speaking voice and occasionally dysphagia means that physicians continue to seek alternative treatments to total laryngectomy, according to our opinion. Certain advanced types of cancer that are managed with partial laryngectomy or primary radiotherapy (with preservation of laryngeal 
function) may achieve results similar to those of total laryngectomy. However, primary radiotherapy is associated with lower patient survival rates, particularly in more advanced disease states, although salvage total laryngectomy remains a backup in cases of radiotherapy failure (4). The results of several studies suggest that patients with advanced cancer of the larynx or hypopharynx with tumor regression following neoadjuvant systemic cytostatic chemotherapy and subsequent radiotherapy may be expected to have improved survival rates, compared with those that undergo radiotherapy alone $(5,6)$.

In 1985, the Veterans Affairs Medical Center conducted a landmark prospective, multicenter, randomized study (The VA Study) to determine whether neoadjuvant chemotherapy followed by radiotherapy (with salvage total laryngectomy in the case of treatment failure) was a more appropriate approach than total laryngectomy with post-operative radiotherapy for patients with advanced stage III and IV laryngeal cancer (1). The results revealed that, to preserve the larynx without reducing overall patient survival, neoadjuvant chemotherapy followed by radiotherapy may be effective for many patients with advanced laryngeal cancer. This previous study initiated a series of preservation protocols, which confirmed that neoadjuvant chemotherapy followed by radiotherapy was an effective alternative treatment for advanced laryngeal cancer (7). As a result, there was a subsequent reduction in the number of total laryngectomies performed (8).

A second study, the Radiation Therapy Oncology Group (RTOG) 91-11 study, was led by the RTOG and the Head and Neck Intergroup. It aimed to determine the efficacy of chemotherapy and radiotherapy in larynx-preserving treatments for advanced laryngeal cancer (2). The RTOG 91-11 study compared the effects of radiotherapy alone, neoadjuvant chemotherapy followed by radiotherapy and concomitant chemoradiotherapy. All treatment modalities were revealed to result in comparable overall patient survival rates, with concomitant chemoradiotherapy determined to be the most likely to preserve the larynx. As such, concomitant chemoradiotherapy became the primary alternative to total laryngectomy in the treatment of advanced laryngeal cancer. Subsequently there was a decline in the use of primary total laryngectomy treatment, and a shift toward reserving it for salvage procedures in cases where primary non-surgical treatments failed (9).

The present study analyzed treatment results of advanced laryngeal cancer patients at the Royal Vinohrady Teaching Hospital (RVTH) to assess whether they were consistent with the aforementioned observations. The primary objective of the current study was not to determine the percentage of laryngeal preservations, but to determine what effect a departure from primary total laryngectomy, for advanced laryngeal cancer, would have on overall patient survival.

\section{Materials and methods}

Patients. Between January 1997 and December 2013, the Charles University 3rd Medical School and RVTH Ear, Nose and Throat (ENT) oncology team (Prague, Czech Republic) treated 185 patients (30 females, 155 males; mean age, $60.7 \pm 9.4$ years; age range, 40-86 years) with advanced laryngeal cancer, which (from a surgical perspective) required total laryngectomy. Written informed consent was obtained from all patients prior to enrollment in the present study and the study was approved by The Ethics Committee of The Medical Faculty of Charles University. Patients with inoperable disease or with poor overall health who were not candidates for surgical treatment were excluded from the study. None of the patients in the present sample group had tumors that were manageable with partial laryngectomy. Any patient that had previously treated head and neck cancer, or any duplicity, were also excluded. Inclusion criteria were operable laryngeal cancer between T2-T4 stages with a Karnofsky index $>60 \%$ (10). In this retrospective analysis, the sample was divided into patients who underwent total laryngectomy and post-operative radiotherapy, and those who were treated conservatively without primary surgery (for example by radiotherapy, neoadjuvant systemic cytostatic chemotherapy followed by radiotherapy, or concomitant chemoradiotherapy). Inclusion in the surgical and non-surgical arms of the present study was primarily based on the personal preference of the patient.

Patient analysis. Overall, $70 \%$ of the patients $(n=129)$ had undergone conventional surgery [total laryngectomy with post-operative radiotherapy (TL)], and $\sim 30 \%(n=56)$ had been treated with larynx preservation protocols (PP). The majority of patients had extensive T4 tumors (41.6\%) and stage IV disease (51.9\%; Table I), as classified using the 2009 Union for International Cancer Control Tumor Node Metastasis Classification of Malignant tumors 7th edition (3).

Statistical analysis. The following statistical methods were used: The nonparametric Mann-Whitney U-test was used to compare the effect of age on treatment, the $\chi^{2}$ test of independence was used to analyze the characteristics of the patient population (extent, stage of disease, sex, local recurrence and nodal recurrence). Kaplan-Meier survival analysis with the Mantel-Cox test of survival functions between categories was performed. The data was presented as the mean \pm standard deviation. SPSS 13.0 statistical software (SPSS, Inc., Chicago, IL, USA) was used for all statistical analysis. $\mathrm{P}<0.05$ was considered to indicate a statistically significant difference.

\section{Results}

A total of 28 patients exhibited local recurrence after treatment (15.1\%). Of these 28, 11 local recurrences were identified in primary surgical TL patients $(8.5 \%)$, and $17 / 28$ recurrences were present in primary non-surgical PP patients $(30.4 \%)$. In total, 14 patients who received preservation treatment $(25 \%)$ underwent a salvage total laryngectomy due to failed primary non-surgical treatment. Cervical nodal recurrence was observed in 10 patients (5.4\% of treated patients), of whom $6(4.7 \%)$ were TL patients and $4(7.1 \%)$ PP patients.

Sex and age. The TL and PP groups were examined in an effort to identify dependencies and differences among specified parameters (sex, age, tumor extent, disease stage, local recurrence, cervical nodal recurrence and 5-year survival rate). Primary surgery had been performed on $56.7 \%$ of the females, and on $72.3 \%$ of the males in the present study. Male patients 
Table I. Patient sample characteristics.

\begin{tabular}{|c|c|c|c|c|c|}
\hline Variables & Total, n (\%) & Primary surgical treatment, n (\%) & No surgery, n (\%) & $\chi^{2}$-value & P-value \\
\hline Extent & & & & 37.690 & $0.001^{\mathrm{a}}$ \\
\hline $\mathrm{T} 2$ & $38(20.5)$ & $14(10.8)$ & $24(42.9)$ & & \\
\hline $\mathrm{T} 3$ & $70(37.8)$ & $45(34.9)$ & $25(44.6)$ & & \\
\hline $\mathrm{T} 4$ & $77(41.6)$ & $70(54.3)$ & $7(12.5)$ & & \\
\hline Stage & & & & 42.124 & $0.001^{\mathrm{a}}$ \\
\hline II & $28(15.1)$ & $7(5.4)$ & $21(37.5)$ & & \\
\hline III & $61(33.0)$ & $38(29.5)$ & $23(41.1)$ & & \\
\hline IV & $96(51.9)$ & $84(65.1)$ & $12(21.4)$ & & \\
\hline Sex & & & & 1.739 & 0.098 \\
\hline Female & $30(16.2)$ & $17(13.2)$ & $13(23.2)$ & & \\
\hline Male & $155(83.8)$ & $112(86.8)$ & $43(76.8)$ & & \\
\hline Local recurrence & & & & 15.422 & $0.001^{\mathrm{a}}$ \\
\hline No & $157(84.9)$ & $118(91.5)$ & $39(69.6)$ & & \\
\hline Yes & $28(15.1)$ & $11(8.5)$ & $17(30.4)$ & & \\
\hline Cervical nodal recurrence & & & & 0.423 & 0.515 \\
\hline No & $175(94.6)$ & $123(95.3)$ & $52(92.9)$ & & \\
\hline Yes & $10(5.4)$ & $6(4.7)$ & $4(7.1)$ & & \\
\hline
\end{tabular}

${ }^{\mathrm{a}} \mathrm{P}<0.05$.

underwent surgery more frequently and this difference was not statistically significant $(\mathrm{P}<0.100$; Table $\mathrm{I})$. The mean age of PP patients was significantly higher than that of TL patients (66 vs. 59 years, respectively; $\mathrm{P}<0.001$; Table II).

Tumor extent and disease stage. Patients with relatively extensive tumors were more frequently indicated for primary surgical treatment, a finding that was statistically significant $(\mathrm{P}<0.001)$. In total, $54.3 \%$ of TL patients had T4 tumors, whereas $12.5 \%$ of PP patients had T4 tumors (Table I). There was also a difference between the groups with respect to disease stage. Stage IV disease was the diagnosis of $21.4 \%$ of PP patients, compared with $65.1 \%$ of TL patients. This difference was statistically significant $(\mathrm{P}<0.001$; Table I).

Local and cervical nodal recurrence. Primary non-surgical patients had a significantly higher frequency of locally recurrent laryngeal cancer $(\mathrm{P}<0.001)$. Only $8.5 \%$ of TL patients experienced local recurrence, compared with $30.4 \%$ of PP patients (Table I). The two patient groups had a low frequency of recurrent cervical node metastases, and no statistically significant differences were recorded relative to this parameter (Table I).

Survival analyses. Kaplan-Meier survival analyses revealed that PP patients had a 5-year survival probability of $\sim 48 \%$, whereas TL patients had a 5-year survival probability of $\sim 63 \%$ (Fig. 1). No statistically significant difference was observed between the 2- and 5-year survival rates (at the $\mathrm{P}<0.050$ significance level), regardless of whether patients underwent primary surgical or non-surgical treatment. TL patients exhibited a statistically significant longer survival $(\mathrm{P}<0.010)$ compared with PP patients.
Table II. Patient sample characteristics with respect to age.

\begin{tabular}{lcl}
\hline Surgical treatment $^{\mathrm{a}}$ & Patients, $\mathrm{n}$ & Age, years $^{\mathrm{b}}$ \\
\hline No & 56 & $65.68 \pm 11.02$ \\
Yes & 129 & $58.58 \pm 7.76$ \\
\hline
\end{tabular}

${ }^{\mathrm{a}} \mathrm{U}=2242.0, \mathrm{P}<0.001$ by the Mann-Whitney U-test. ${ }^{\mathrm{b}}$ Mean \pm standard deviation.

An overview of survival analyses in association with the studied parameters (sex, age, tumor extent, disease stage, local recurrence and cervical nodal relapse) is presented for TL and PP patients in Tables III and IV, respectively. The data demonstrate that survival is particularly affected by local and cervical node disease recurrence in TL patients $(\mathrm{P}<0.001)$, and by recurrent local carcinoma in PP patients $(\mathrm{P}<0.100)$. Kaplan-Meier survival analyses confirmed that sex did not affect the probability of survival. Male and female patients treated for laryngeal cancer that, according to standard indications, required total laryngectomy had a 5-year survival probability of $\sim 60 \%$, irrespective of treatment approach.

Patients $>71$ years of age had a significantly lower 5-year survival probability $(\sim 35 \%)$ than younger patients $(>60 \%$; $\mathrm{P}<0.010)$. The highest 5-year survival probability was observed in patients with the least extensive tumors (T2, $\leq 75 \%$; T3, $\sim 62 \%, \mathrm{~T} 4, \sim 50 \%$ ); however, these differences were not statistically significant. Disease stage also affected 5-year survival probability. Irrespective of the treatment method, 5-year survival probability was $\sim 75 \%$ in patients with stage II, $65 \%$ 
in patients with stage III, and 55\% in patients with stage IV disease; however, these differences were not statistically significant.

Local disease recurrence had a marked effect on 5-year survival probability. Regardless of treatment approach, patients without local recurrence had a 5-year survival probability of $\sim 70 \%$, whereas patients with local recurrence had a 5-year survival probability of only $20 \%$, a significant difference $(\mathrm{P}<0.001)$. Recurrent cervical node metastases had a major effect on patient survival. Irrespective of treatment method, laryngeal cancer patients without cervical node recurrence had a 5-year survival probability of $65 \%$, where as that of patients with cervical nodal recurrence was $<20 \%$, a significant difference $(\mathrm{P}<0.001)$.

An evaluation of the entire patient cohort indicated that the treatment method affected survival probability, and that TL patients survived significantly longer $(\mathrm{P}<0.010)$. The median survival time (the period during which at least $50 \%$ of patients are alive) was 97 months ( $>8$ years) for TL patients, but only 56 months $(<5$ years) for PP patients. Survival probabilities were significantly different for various age groups (patients $>70$ years survived the treatments for a significantly shorter period; $\mathrm{P}<0.010$ ), those that exhibited local recurrence vs. those that did not $(\mathrm{P}<0.001)$ and those that exhibited cervical node recurrence vs. those that did not $(\mathrm{P}<0.001$; Table V).

To determine which of the chosen parameters most significantly affected survival, stepwise Cox regression analysis was used to examine sex, patient age, tumor extent, disease stage, choice of primary treatment, local recurrence and cervical node recurrence. From these analyses, recurrence was selected at the first step as the parameter that most affected survival $(\mathrm{P}<0.001)$, age $>70$ years was selected at the second step $(\mathrm{P}<0.001)$, cervical node recurrence was selected at the third step $(\mathrm{P}<0.001)$ and disease stage was selected at the fourth step $(\mathrm{P}<0.010)$. The remaining parameters did not have a statistically significant impact on survival.

\section{Discussion}

The analyses of the present study differed fundamentally from the aforementioned VA and RTOG 91-11 studies $(1,2)$, in that the current study was not a prospective randomized multicenter one, but rather a retrospective study based on the experience of one center. The present cohort included patients with laryngeal cancer who, from a surgical view point, would normally require total laryngectomy. Overall patient health and disease extent governed the recommendations of the ENT oncology team (i.e., T4 tumors were recommended for surgical management). As such, the patient representation in the two study groups contained a bias that would be in admissible in a prospective randomized study.

Patient samples. The present study included 185 patients with advanced cancer; 129 of these patients underwent primary surgical treatment and 56 underwent primary non-surgical treatment in an effort to preserve the larynx. The VA study was based on an evaluation of 332 patients; $\sim 50 \%$ of these patients were in the surgical group (i.e., treated with total laryngectomy and post-operative radiotherapy), while the remaining half were assigned to the

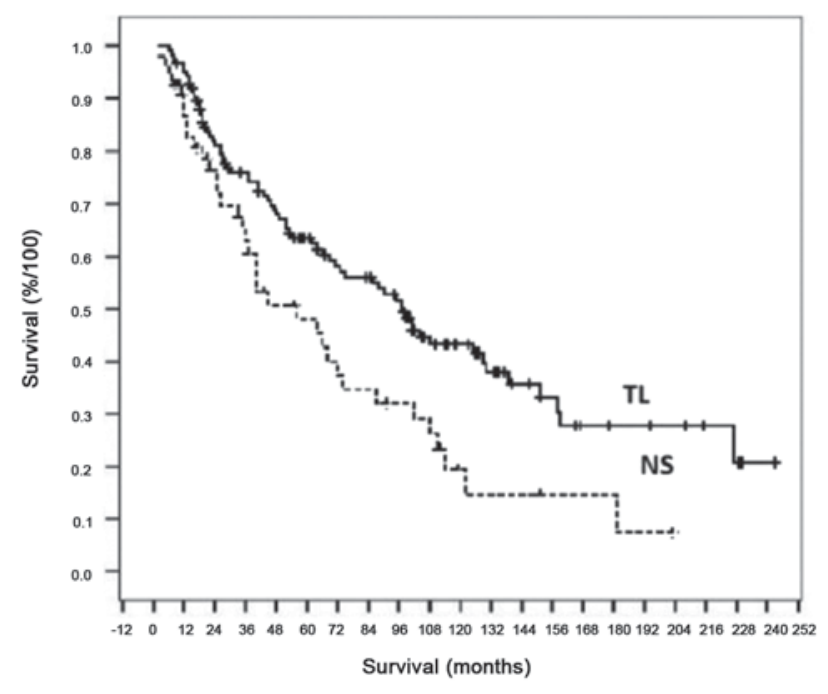

Figure 1. Kaplan-Meier survival analysis with Mantel-Cox test curves for patients treated with TL and patients with NS. TL 5-year survival probability was $63 \%$ and NS 5 -year survival probability was $48 \%$. TL, total laryngectomy; NS, primary non-surgical treatment. Mantel-Cox test: Chi-square $=7.585, \mathrm{P}=0.006$.

non-surgical group. The primary non-surgical treatment group of the present study consisted of patients treated with: i) Neoadjuvant systemic chemotherapy according to the VA study protocol; ii) patients treated with concomitant chemoradiotherapy according to the RTOG 91-11 study protocol; iii) patients treated with radiotherapy alone. The merging of these different patient groups into one was based on the outcome of the RTOG 91-11 study, which identified no statistically significant difference in overall survival between the three types of treatment. The RTOG 91-11 study evaluated 518 patients.

The mean patient age in the present study was 60.7 years, with a median age of 60 years. This was very similar to the mean patient ages in the VA study (mean age, 62 years) and in the RTOG 91-11 study, which had a median age of 59 years for patients treated with radiotherapy or neoadjuvant chemotherapy, and a median age of 60 years for patients treated with concomitant chemoradiotherapy. In contrast to these two randomized prospective studies, the present RVTH patient sample had a statistically significant age difference between patients in the surgical (mean age, 58.6 years) and non-surgical (mean age, 65.7 years) treatment groups. Thus, younger patients were more often recommended for surgery.

Regarding gender distribution, the present study included 155 males (84\%) and 30 females (16\%), the VA study included 321 males (97\%) and 11 females (3\%) and the RTOG 91-11 study included 401 males (77\%) and 117 females (23\%). The VA study had a significantly lower representation of female patients. Given that no statistically significant difference in the overall survival of males and females was identified in the RVTH sample, the variation in the number of males and females among the studies was not considered significant.

The inclusion criteria for the VA study protocol included stage III or IV laryngeal cancer with a Karnofsky index of $>50$. Patients with T1N1 tumors and inoperable tumors were excluded, as were patients with distant metastasis or a history 
Table III. Kaplan-Meier survival analysis of the primary surgical treatment group and the Mantel-Cox test between categories of individual, and possibly dependent, parameters.

\begin{tabular}{|c|c|c|c|c|c|c|}
\hline Group & Patients, n & Mortalities, $\mathrm{n}$ & Survival Rate, $\%$ & Median & Mantel-Cox Z-value & P-value \\
\hline Sex & & & & & 1.286 & 0.257 \\
\hline Female & 17 & 6 & 64.7 & 158.0 & & \\
\hline Male & 112 & 64 & 42.9 & 95.0 & & \\
\hline Age, years & & & & & 3.828 & 0.148 \\
\hline$\leq 50$ & 20 & 12 & 40.0 & 108.0 & & \\
\hline $51-70$ & 99 & 49 & 50.5 & 99.0 & & \\
\hline$>70$ & 10 & 8 & 20.0 & 47.0 & & \\
\hline Tumor extent & & & & & 2.791 & 0.248 \\
\hline $\mathrm{T} 2$ & 14 & 6 & 57.1 & 158.0 & & \\
\hline $\mathrm{T} 3$ & 45 & 24 & 46.7 & 101.0 & 7.317 & $0.026^{\mathrm{a}}$ \\
\hline $\mathrm{T} 4$ & 70 & 39 & 44.3 & 71.0 & & \\
\hline Disease stage & & & & & 3.020 & 0.221 \\
\hline II & 7 & 3 & 57.1 & 158.0 & & \\
\hline III & 38 & 20 & 47.4 & 125.0 & 7.110 & $0.026^{\mathrm{a}}$ \\
\hline IV & 84 & 46 & 45.2 & 75.0 & & \\
\hline Local recurrence & & & & & 35.125 & $0.001^{\mathrm{a}}$ \\
\hline No & 118 & 59 & 50.0 & 101.0 & & \\
\hline Yes & 11 & 10 & 9.1 & 14.0 & & \\
\hline Cervical nodalrecurrence & & & & & 46.474 & $0.001^{\mathrm{a}}$ \\
\hline No & 123 & 63 & 48.8 & 101.0 & & \\
\hline Yes & 6 & 6 & 0.0 & 14.0 & & \\
\hline
\end{tabular}

${ }^{\mathrm{a}} \mathrm{P}<0.05$

of prior radiotherapy to the head and neck region. The majority of patients had stage III supraglottic cancer.

None of the patients in the present sample group had tumors that were manageable with partial laryngectomy, unlike the VA study. The non-surgical study arm of the current study was mostly represented by patients with stage III cancer and T3 tumor extension. Of the surgically treated patients, the majority had stage IV cancer with T4 extension. This reflects the strategy to recommend patients with advanced tumors for surgery. In contrast to the VA study, the present patient sample also included patients at stage II with T2 tumors (particularly in the non-surgical group). This stems from the understanding that even T2 laryngeal tumors can sometimes require surgical management with total laryngectomy. Furthermore, the RVTH ENT oncology team strictly adhered to the Union for International Cancer Control (UICC) recommendations. Therefore, if doubts exist regarding the TNM classification, the lower category (less advanced) is selected (6). It is, therefore, possible that a large number of $\mathrm{T} 2$ laryngeal cancer cases in the present patient cohort may have been classified as T3 at a different treatment center.

The majority of patients had T3 supraglottic cancer without vocal cord fixation (N0, stage III) in the RTOG 91-11 study. The RTOG 91-11 study also had the inclusion criterion of stage III or IV laryngeal cancer with a Karnofsky index of $>60$, and excluded synchronous duplicate tumors. Tumors with T1 extension and (unlike the present patient sample) T4 tumors with extension through the thyroid cartilage or a $>1 \mathrm{~cm}$ extension into the base of the tongue were excluded.

Study outcomes. In the VA study non-surgical treatment group, $64 \%$ of patients had a preserved larynx two years following treatment. Total laryngectomy was required in $7 \%$ of patients owing to late tumor recurrence ( $>3$ months after treatment), $80 \%$ of which occurred during the first-year post-treatment. Salvage laryngectomy was performed in $29 \%$ of patients owing to disease persistence following chemotherapy, or within three months of the conclusion of radiotherapy. A greater frequency of local recurrence was observed in tumors with glottis localization, tumors with vocal cord fixation, and thyroid cartilage invasion; however, these factors did not affect the statistical significance of local recurrence. Salvage total laryngectomy was performed significantly more often in stage IV cancer cases with a T4 extension. Subsequent stand-alone block dissection was only performed in 10 patients. Local disease recurrence occurred significantly less often in the surgical study arm ( 2 vs. $12 \%$ in the non-surgical arm, $\mathrm{P}<0.001$ ). Cervical node recurrence was observed in $5 \%$ of patients in the surgical arm and in $8 \%$ of patients in the chemotherapy groups, though this difference was not statistically significant.

Similarly, local disease recurrence occurred statistically significantly more often in the non-surgical treatment group of the present study (30.4 vs. $8.5 \%$; $\mathrm{P}<0.001)$, although in contrast to the VA study, the division of tumor extension and 
Table IV. Kaplan-Meier survival analysis of the laryngeal cancer patients in the primary non-surgical treatment group and the Mantel-Cox test between categories of individual, possibly dependent, parameters.

\begin{tabular}{|c|c|c|c|c|c|c|}
\hline Group & Patients, $\mathrm{n}$ & Mortalities, $n$ & Survival rate, $\%$ & Median & Mantel-CoxZ-value & P-value \\
\hline Sex & & & & & 0.203 & 0.652 \\
\hline Female & 13 & 7 & 46.2 & 45.0 & & \\
\hline Male & 43 & 30 & 30.2 & 56.0 & & \\
\hline Age, years & & & & & 2.164 & 0.339 \\
\hline$\leq 50$ & 3 & 1 & 66.7 & & & \\
\hline $51-70$ & 33 & 20 & 39.4 & 64.0 & & \\
\hline$>70$ & 20 & 16 & 20.0 & 40.0 & & \\
\hline Tumor extent & & & & & 3.887 & 0.143 \\
\hline $\mathrm{T} 2$ & 24 & 13 & 45.8 & 68.0 & & \\
\hline $\mathrm{T} 3$ & 25 & 19 & 24.0 & 40.0 & & \\
\hline $\mathrm{T} 4$ & 7 & 5 & 28.6 & 25.0 & & \\
\hline Disease stage & & & & & 2.831 & 0.243 \\
\hline II & 21 & 12 & 42.9 & 68.0 & & \\
\hline III & 23 & 17 & 26.1 & 40.0 & & \\
\hline IV & 12 & 8 & 33.3 & 56.0 & & \\
\hline Local recurrence & & & & & 3.624 & 0.057 \\
\hline No & 39 & 23 & 38.5 & 66.0 & & \\
\hline Yes & 17 & 13 & 23.5 & 25.0 & & \\
\hline Cervical nodal recurrence & & & & & 1.515 & 0.218 \\
\hline No & 52 & 34 & 34.6 & 56.0 & & \\
\hline Yes & 4 & 3 & 25.0 & 19.0 & & \\
\hline
\end{tabular}

stage was not symmetrical (i.e., the surgical group had more patients with T4 tumors and stage IV disease). This implies that surgical management has an unambiguously positive impact in the prevention of local recurrence. In total, 17 patients in the non-surgical treatment group of the present study had local recurrences, of which $14(82.4 \%)$ underwent a salvage total laryngectomy. Of the remaining three patients, one patient refused salvage laryngectomy and two were inoperable owing to the overall poor health of the patients (one patient had suffered an acute myocardial infarction, and one patient had suffered a severe cerebrovascular accident). Cervical node recurrence was diagnosed in $4.7 \%$ of the patients in the primary surgical treatment group, and in $7.1 \%$ of the primarily non-surgical treatment group, which was not a statistically significant difference. As with the VA study, $\sim 66 \%$ of non-surgical treatment patients in the present study had a preserved larynx. In contrast to the VA study, however, one-third of the patients of the present study had stage II disease or, more precisely, were borderline between stage II and III. However, the non-surgical approach was burdened by a higher risk of recurrence.

The RTOG 91-11 study, which compared the efficacy of non-surgical treatment for the entire sample, achieved a 2-year patient survival rate of $65 \%$ without total laryngectomy. The 2 -year survival rates with larynx preservation were: $88 \%$ in patients treated with concomitant chemoradiotherapy; $75 \%$ in patients treated with induction chemotherapy; $70 \%$ in patients treated with radiotherapy alone. Successful concomitant chemoradiotherapy was significantly better for laryngeal preservation than induction chemotherapy or radiotherapy alone.
In terms of larynx preservation, no significant difference was observed between the efficacy of induction chemotherapy and radiotherapy alone. The conclusions of the RTOG 91-11 study demonstrated that primary non-surgical treatment may save the larynx in $\sim 66 \%$ of patients with locally-advanced laryngeal cancer. In this respect, concomitant chemoradiotherapy is the most efficacious approach. Since induction chemotherapy does not produce (in terms of laryngeal preservation) better results than radiotherapy alone, if chemoradiotherapy is not possible then radiotherapy alone without chemotherapy is indicated.

Analysis and comparison of the studies. The fundamental parameter of cancer treatment assessed in the present study was overall patient survival. Analyses of the patient sample of the present study indicate that treatment method significantly affects patient survival. TL patients survived significantly longer than patients with PP patients $(\mathrm{P}<0.010)$. The 2-year survival probability of PP patients was 75 vs. $82 \%$ in TL patients and the 5-year survival probability was $48 \%$ in PP patients vs. $63 \%$ in TL patients. However, the difference between 2- and 5-year survival rates was not determined to be significant in either group. In TL patients, survival was affected by the pre-operative parameters of tumor extent and disease stage $(\mathrm{P}<0.05)$, as well as the post-operative parameters of local recurrence and cervical node recurrence $(\mathrm{P}<0.001)$. In PP patients, none of the pre-treatment parameters (sex, age, tumor extent and disease stage) were determined to significantly affect survival. The post-treatment parameter local recurrence was revealed to have a no statistically significant impact $(\mathrm{P}<0.100)$. 
Table V. Complete overview: Kaplan-Meier survival analysis for the entire patient sample and the Mantel-Cox test between categories of individual, possibly dependent, parameters.

\begin{tabular}{|c|c|c|c|c|c|c|}
\hline Group & Patients, $\mathrm{n}$ & Mortalities, $\mathrm{n}$ & Survival Rate, $\%$ & Median & Mantel-Cox Z-value & P-value \\
\hline Surgical treatment & & & & & 7.585 & 0.006 \\
\hline No & 56 & 37 & 33.9 & 56.0 & & \\
\hline Yes & 129 & 70 & 45.7 & 97.0 & & \\
\hline Sex & & & & & 0.553 & 0.457 \\
\hline Female & 30 & 13 & 56.7 & 122.0 & & \\
\hline Male & 155 & 94 & 39.4 & 74.0 & & \\
\hline Age & & & & & 10.244 & 0.006 \\
\hline$\leq 50$ & 23 & 13 & 43.5 & 108.0 & & \\
\hline $51-70$ & 132 & 69 & 47.7 & 90.0 & & \\
\hline$>70$ & 30 & 24 & 20.0 & 45.0 & & \\
\hline Tumor extent & & & & & 1.752 & 0.416 \\
\hline $\mathrm{T} 2$ & 38 & 19 & 50.0 & 111.0 & & \\
\hline $\mathrm{T} 3$ & 70 & 43 & 38.6 & 88.0 & & \\
\hline $\mathrm{T} 4$ & 77 & 45 & 41.6 & 54.0 & & \\
\hline Disease stage & & & & & 1.038 & 0.595 \\
\hline II & 28 & 15 & 46.4 & 111.0 & & \\
\hline III & 61 & 37 & 39.3 & 88.0 & & \\
\hline IV & 96 & 55 & 42.7 & 62.0 & & \\
\hline Local recurrence & & & & & 38.656 & 0.001 \\
\hline No & 157 & 82 & 47.8 & 99.0 & & \\
\hline Yes & 28 & 24 & 14.3 & 24.0 & & \\
\hline Cervical node recurrence & & & & & 28.399 & 0.001 \\
\hline No & 175 & 97 & 44.6 & 90.0 & & \\
\hline Yes & 10 & 9 & 10.0 & 15.0 & & \\
\hline
\end{tabular}

Analysis of the entire patient sample of the current study identified that pre-treatment parameters, treatment method and patient age, had a significant impact on the survival of patients with advanced laryngeal cancer $(\mathrm{P}<0.010)$; however, the post-treatment parameters local and cervical node recurrence were even more significant $(\mathrm{P}<0.001)$. Analyses of the patient samples of the current study suggested that primary non-surgical treatment could be used to save the larynx in $\sim 66 \%$ of patients, but it is burdened with a higher risk of local recurrence, and negatively impacts patient survival $(\mathrm{P}<0.01)$; however, it did not statistically significantly affect the 2- and 5-year survival rates.

In the VA study, the 2-year survival probability for total laryngectomy patients and primary non-surgical treatment patients was identical (68\%) and therefore was similar to (or slightly worse than) the sample of the current study (82 and $75 \%$, respectively). In the RTOG 91-11 study, the 2-year survival probability was $76 \%$ for patients in the induction chemotherapy group, $74 \%$ in the chemoradiotherapy group and $75 \%$ in the radiotherapy alone group. The respective 5 -year survival probabilities for these groups were 55, 54 and $56 \%$, with no significant difference between them. These results are comparable with the previous two samples. Analysis from the RTOG 91-11 study revealed that none of the primary conservative treatment methods improved overall patient survival and that the incorporation of chemotherapy improved larynx preservation only in form chemoradiotherapy. Since induction chemotherapy did not increase likelihood of larynx preservations compared with radiotherapy alone, the method of choice was to include chemotherapy as part of a chemoradiotherapy regimen. On the basis of the results of the present study, platinum-based induction chemotherapy alone is not appropriate.

Analyses of the sample of the present study confirmed the conclusions of the VA and RTOG 91-11 pilot studies, which identified that primary non-surgical treatment of advanced laryngeal cancer may save the larynx in $\sim 66 \%$ of patients. However, the present analyses also confirmed that primary non-surgical treatment has a significantly higher risk of local recurrence. Although no statistically significant difference was observed between 2 and 5-year survival rates (in agreement with the VA study), primary non-surgical treatment impaired overall patient survival (11). The results of the analysis of the present study must be viewed as the results of a retrospective, non-randomized study. The primary surgical treatment group included younger patients with more extensive tumors and a higher disease stage. However, the present study did confirm that primary non-surgical treatment is an alternative approach to total laryngectomy. Conversely, salvage total laryngectomy is an integral component of the larynx preservation protocol (primary non-surgical treatment of advanced resectable laryngeal cancer). The final decision regarding treatment 
approach should be left to the patient. Nonetheless, the reduced patient survival rate, particularly for more extensive tumors and in younger individuals, means that primary surgical treatment should be recommended in similar cases. If a patient opts for primary non-surgical treatment, the indicated treatment is concomitant chemoradiotherapy, which is currently based on the results of the RTOG 91-11 study. If a patient is unable to undergo chemotherapy, they should undergo radiotherapy alone; the administration of platinum-based systemic cytostatic induction chemotherapy is not considered justifiable. However, there is little doubt that the addition of novel cytostatic drugs such as taxanes and biological therapies such as cetuximab to induction chemotherapy may lead to its renaissance (12). The toxicity of platinum-based chemoradiotherapy is substantial and many patients are unable to complete the prescribed chemotherapy or radiotherapy treatments. It is likely that primary non-surgical treatment combinations will vary in the future, depending on results from new, randomized, multicenter, prospective studies such as EORTC 24954 or TREMPLIN (13-15).

When deciding on treatment methods, it is also necessary to consider that, although primary non-surgical treatment approach can save the larynx in $\sim 66 \%$ of patients, the preserved larynx may not be fully functional. Some patients with laryngeal preservation following primary non-surgical treatment who do not present with loco regional recurrence may nonetheless require tracheostomy, or even gastrostomy, for dysphagia. This reality adds further complications to the choice of laryngeal preservation protocols (16).

A patient sample treated by the RVTH ENT oncology team between 1997 and 2013 was divided into those that received conventional treatment (i.e., total laryngectomy and post-operative radiotherapy), and those treated with laryngeal preservation protocols (i.e., chemoradiotherapy, neoadjuvant cytostatic chemotherapy followed by radiotherapy, or radiotherapy alone, and, in the event of non-surgical treatment failure, possible subsequent total laryngectomy). The aim of the present study was to compare both groups in an effort to prove or refute the findings of the VA and RTOG 91-11 pilot studies: That primary non-surgical treatment of locally advanced laryngeal cancer does not reduce the likelihood of patient survival, and may preserve a functional larynx in $\sim 66 \%$ of patients. The 2 -year survival probability of primary non-surgical patients was $75 \%$, and the 5-year survival probability was $48 \%$. In primary surgical patients, the 2 -year survival probability was $82 \%$ and the 5 -year survival probability was 63 . However, this difference was not identified to be statistically significant. The present study confirmed that primary non-surgical treatment can save the larynx in approximate two-thirds of patients, but also identified that primary non-surgical treatment is burdened with a higher risk of local recurrence and a poorer overall survival rate $(\mathrm{P}<0.010)$.

In conclusion, the current study confirmed that primary non-surgical treatment is an alternative approach to total laryngectomy. Conversely, salvage total laryngectomy remains an integral component of laryngeal preservation protocols. The final decision regarding treatment approach should be left to the informed patient. A decreased overall survival rate, particularly in more extensive tumors and in younger patients, means that surgical treatment is recommended in these cases. If a patient opts for primary non-surgical treatment, it should be based on the RTOG 91-11 study results, which call for concomitant chemoradiotherapy. If the patient is unable to undergo cytostatic chemotherapy, radiotherapy alone should be used. The administration of platinum-based systemic cytostatic induction chemotherapy is not considered justified.

\section{References}

1. The Department of Veterans Affairs Laryngeal Cancer Study Group, Wolf GT, Fisher SG, Hong WK, Hillman R, Spaulding M, Laramore GE, Endicott JW, McClatchey K and Henderson WG: Induciton chemotherapy plus radiation compared with surgery plus radiation in patients with advanced laryngeal cancer. $N$ Engl J Med 324: 1685-1690, 1991.

2. Forastiere AA, Goepfert H, Maor M, Pajak TF, Weber R, Morrison W, Glisson B, Trotti A, Ridge JA, Chao C, et al: Concurrent chemotherapy and radiotherapy for organ preservation in advanced laryngeal cancer. N Engl J Med 349: 2091-2098, 2003.

3. International Union Against Cancer (UICC): TNM Classification of Malignant Tumours. Sobin LH, Gospodarowicz MK and Wittekind C (eds). 7th edition. Wiley-Blackwell, New York, NY, 2009.

4. Harris HS Jr, Watson FR and Spratt JS Jr: Carcinoma of the larynx: A retrospective study of 144 cases. Am J Surg 118: 674-684, 1969.

5. Demard F, Chauvel P, Santini J, Vallicioni J, Thyss A and Schneider M: Response to chemotherapy as justification for modification of the therapeutic strategy for pharyngolaryngeal carcinomas. Head Neck 12: 225-231, 1990.

6. Karp D, Carter R and Vaughan C: Voice preservation using induction chemotherapy plus radiation therapy as an alternative to laryngectomy in advanced head and neck cancer: Long term follow-up. Proc Am Soc Clin Oncol 7: 152, 1988.

7. Forastiere AA, Weber RS and Trotti A: Organ preservation for advanced larynx cancer: Issues and outcomes. J Clin Oncol 33: 3262-3268, 2015.

8. Klozar J, Cada Z and Koslabova E: Complications of total laryngectomy in the era of chemoradiation. Eur Arch Otorhinolaryngol 269: 289-293, 2012.

9. Weber RS, BerkeyBA Forastiere A, Cooper J, Maor M, Goepfert H, Morrison W, Glisson B, Trotti A, Ridge JA, et al: Outcome of salvage total laryngectomy following organ preservation therapy: The radiation therapy oncology group trial 91-11. Arch Otolaryngol Head Neck Surg 129: 44-49, 2003.

10. Karnofsky David A, Abelmann WH, Craver LF and Burchenal Joseph H: The use of the nitrogen mustards in the palliative treatment of carcinoma-with particular reference to bronchogenic carcinoma. Cancer 1: 634-656, 1948.

11. Megwalu UC and Sikora AG: Survival outcomes in advanced laryngeal cancer. JAMA Otolaryngol Head Neck Surg 140: 855-860, 2014.

12. Mesía R, Garcia-Saenz JA, Lozano A, Pastor M, Grau JJ, Martínez-Trufero J, Lambeaz J, Martínez-Galán J, Mel JR, González B, et al: Could the addition of cetuximab to conventional radiation therapy improve organ preservation in those patients with locally advanced larynx cancer who respond to induction chemotherapy? An organ preservation spanish head and neck cancer cooperative group phase 2 study. Int J Radiat Oncol Biol Phys 97: 473-480, 2017.

13. Fayette J, Pointreau Y, Bourhis J and Lefebvre JL: Squamous cell carcinoma of the hypopharynx and larynx: Evidence-based care. Bull Cancer 101: 438-444, 2014 (In French).

14. Lefebvre JL, Pointreau Y, Rolland F, Alfonsi M, Baudoux A, Sire C, de Raucourt D, Malard O, Degardin M, Tuchais C, et al: Induction chemotherapy followed by either chemoradiotherapy or bioradiotherapy for larynx preservation: The TREMPLIN randomized phase II study. J Clin Oncol 31: 853-859, 2013.

15. Lefebvre JL, Rolland F, Tesselaar M, Bardet E, Leemans CR, Geoffrois L, Hupperets P, Barzan L, de Raucourt D, Chevalier D, et al: EORTC head and neck cancer cooperative group; EORTC radiation oncology group: Phase 3 randomized trial on larynx preservation comparing sequential vs. alternating chemotherapy and radiotherapy. J Natl Cancer Inst 101: 142-152, 2009.

16. Theunissen EA, Timmermans AJ, Zuur CL, Hamming-Vrieze O, Paul de Boer J, Hilgers FJ and van den Brekel MW: Total laryngectomy for a dysfunctional larynx after (chemo)radiotherapy. Arch Otolaryngol Head Neck Surg 138: 548-555, 2012. 